\title{
Cluster as current sheet surveyor in the magnetotail
}

\author{
Y. Narita, R. Nakamura, and W. Baumjohann \\ Space Research Institute, Austrian Academy of Sciences, Schmiedlstr. 6, 8042 Graz, Austria \\ Correspondence to: Y. Narita (yasuhito.narita@oeaw.ac.at)
}

Received: 21 August 2013 - Revised: 30 August 2013 - Accepted: 4 September 2013 - Published: 27 September 2013

\begin{abstract}
A novel analysis technique is presented to estimate the current sheet thickness unambiguously and directly, without associating time series data with spatial structure. The technique is a combination of eigenvalue analysis and minimum variance estimator adapted to Harris current sheet geometry, and needs one-time, four-point magnetic field data as provided by the Cluster spacecraft. Two current sheet parameters, thickness and distance to the spacecraft, can be determined at each time step of the magnetic field measurements. An example is shown from a Cluster magnetotail crossing under quiet magnetospheric conditions, yielding the result that the current sheet thickness is on the scale of the proton gyroradius. The analysis technique can also be used to track the dynamical evolution of the current sheet structure in three dimensions.
\end{abstract}

Keywords. Magnetospheric physics (current systems; magnetotail) - space plasma physics (experimental and mathematical techniques)

\section{Introduction}

The magnetotail current sheet has been studied since the earliest era of space physics (Ness, 1965). It is caused by the interaction of solar wind plasma with the Earth's magnetic field, sustaining the stretched field lines along the solar wind flow direction. The current sheet is known to be dynamic, varying in its thickness, orientation, formation, and current density (see reviews by Nakamura et al., 2006 and Zelenyi et al., 2009). It is the place where energy conversion processes such as magnetic reconnection lead to geomagnetic substorms (Baumjohann, 2002). Various models have been proposed to understand the nature of a current sheet in space plasma, e.g., planar geometry, deformed current sheet, or multi-scale or embedded current sheets (Petrukovich et al., 2008, 2011).

Current sheet structure has been studied by various analysis techniques: associating time series data with spatial structure (Zhang et al., 2006), tracing charged particle orbits (Larson and Kaufmann, 1996), and measuring gradient or field rotation using multiple spacecraft (Rong et al., 2011). The potential of multi-spacecraft measurements was fully used to develop, for example, the curlometer technique (e.g., Dunlop et al., 2002), the least-square gradient methods (De Keyser, 2008; Hamrin et al., 2008), and the combination of the curlometer with the discontinuity analyzer (Dunlop and Balogh, 2005) in the studies of current sheet structure and motion. Various methods are also proposed to characterize the current sheet thickness, including minimum variance analysis, the constant velocity approach and the constant thickness approach (Haaland et al., 2004). Current sheet thickness is estimated as being typically one-thousand to several thousands of km (Runov et al., 2006; Zhang et al., 2006), the same order as the proton gyroradius. Reading time series data is most widely used for its easy implementation, giving a reasonable estimate of current sheet thickness under proper assumptions. There is, however, always an ambiguity in reading time series data as a spatial structure, in that the motion and change of the structure during the measurement may influence the time series data as well. In spite of its role in magnetospheric dynamics and its accessibility to us, unambiguous determination of the current sheet structure remains one of the challenges in space plasma research.

In this article, a new analysis technique is constructed particularly for the four-spacecraft Cluster mission (Escoubet et al., 2001). This tool can determine the current sheet thickness and distance to the spacecraft without reading time series data as a spatial structure; It can also be used at a particular time of observation and can be applied to time series data for statistics or the tracking of dynamical evolution. It 


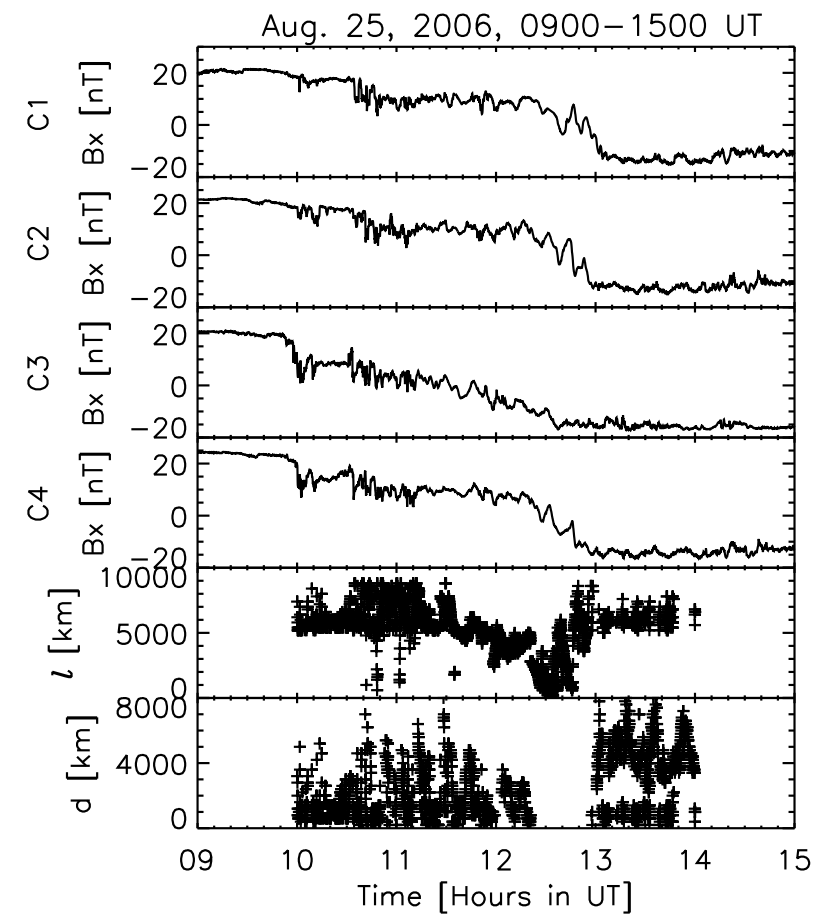

Fig. 1. Time series plots of the sunward component of the magnetic field (GSM- $\left.B_{x}\right)$, the distance between the current sheet and the spacecraft center of mass $\ell$, and the current sheet thickness $d$, as measured by four Cluster spacecraft (using a fluxgate magnetometer).

is a combination of eigenvalue analysis and minimum variance estimator (Haykin, 1991) applied to Harris current sheet geometry (Harris, 1962). The minimum variance estimator has been applied successfully to multi-point magnetic field data. The essence of this estimator is the fact that one can scan the signal power between an arbitrary spatial structure model and measurement data in the parameter space. Spatial structure can be plane wave geometry with three parameters (three components of a wave vector), known as the wave telescope technique (Glassmeier et al., 2001); it can be spherical wave geometry with four parameters (three components of the wave source coordinate and the wavelength), known as the source locator technique (Constantinescu et al., 2006); or it can be a phase-shifted, spatially damped wave (Plaschke et al., 2008). In contrast to those previous applications, the new analysis technique needs only one-time, four-point magnetic field data. Neither a time-stationary condition nor an ergodic hypothesis is needed in the analysis.

\section{Analysis technique applied to Cluster observation}

We apply the minimum variance estimator to the magnetotail crossing of the four Cluster spacecraft on 25 August 2006, 09:00-15:00 UT. This time interval was chosen according to the following criteria. First, Cluster formed a nearly regu- lar tetrahedron, which is suitable for the analysis of spatial structure in three dimensions. The inter-spacecraft distance is about $10000 \mathrm{~km}$. Depending on directions of interest, four sampled fields are available with a typical spacing of 2000$3000 \mathrm{~km}$. Second, the spacecraft apogee is in the midnight sector of late summer (between the end of August and the beginning of September), which is preferable for magnetotail observations. Third, global magnetospheric disturbance is only moderate, associated with the northward interplanetary magnetic field.

Figure 1 (four panels from the top) displays the time series of the sunward component of the magnetic field ( $B_{x}$ in GSM coordinate) measured by the fluxgate magnetometer on board four Cluster spacecraft (Balogh et al., 2001). Cluster crosses the magnetotail from the Northern Hemisphere to the Southern, observing a transition of the magnetic field from sunward $\left(B_{x}>0\right)$ to anti-sunward $\left(B_{x}<0\right)$. Cluster 1,2 , and 4 observe a rather sharp transition temporarily localized between 12:00 and 13:00 UT. Cluster 3 observes, on the other hand, a smooth and slow transition from 10:30 to 12:30 UT. The bottom two panels in Fig. 1 show the results of the analysis: the distance $\ell$ from the current sheet to the spacecraft's center of mass and the current sheet thickness $d$. They are determined from the four-point magnetic field data as a function of time.

The idea of the analysis is as follows. The current sheet model is taken to be of the Harris type, a hyperbolic tangent profile of the magnetic field as a function of the distance of the spacecraft from the current sheet. The model depends on four parameters: two angles to determine the current sheet normal (polar angle $\theta$ and azimuthal angle $\phi$ ), distance $\ell$ from the spacecraft's center of mass to the current sheet along the current sheet normal, and the thickness of the current sheet $d$. The first two parameters (angles) are obtained from the eigenvalue analysis of the four-point magnetic field data by associating the eigenvectors with the current sheet structure. The remaining parameters must be determined by a fitting procedure. To perform the fitting, the minimum variance estimator is used. It computes the covariance between the model magnetic field and the measured field in an adaptive way, minimizing the covariance. The signal power (the output of the estimator) is scanned into the parameter space spanned by the distance $\ell$ and the thickness $d$. Optimal values of the distance and the thickness are obtained by searching for a peak in the signal power distribution.

The detailed analysis consists of three steps: identification of current sheet normal; estimate of current sheet distance and thickness; and evolution analysis of the current sheet.

1. Identification of current sheet normal. Eigenvalue analysis is performed on the three-by-three covariance matrix. Four covariance matrices are constructed from one-time four-point magnetic field data, and are averaged and reduced to one covariance matrix. The Harris current sheet model is constructed in the coordinate 

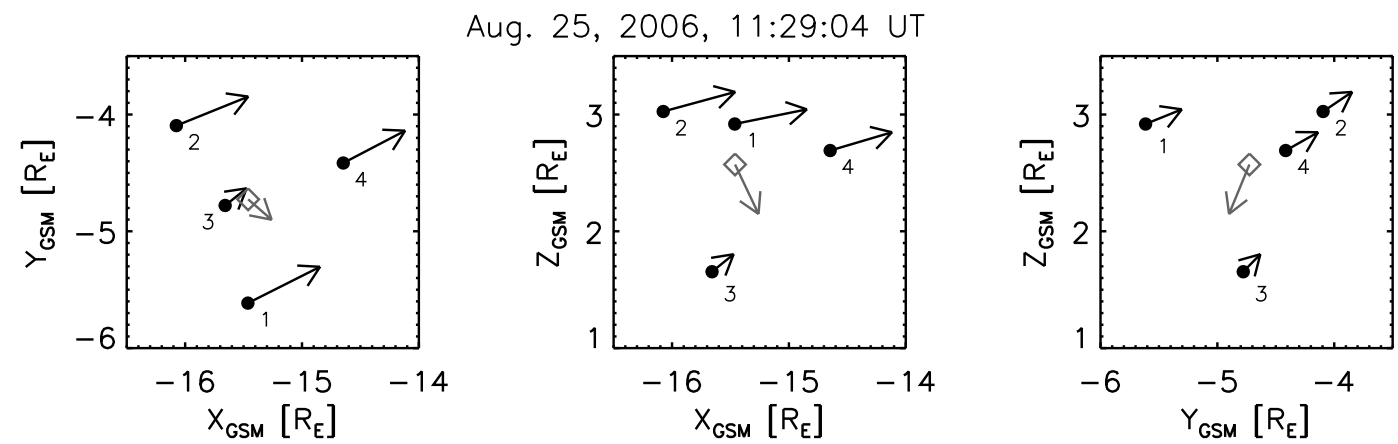

Fig. 2. Projection of magnetic field vectors measured at four Cluster spacecraft (presented as arrows in black) onto GSM $X Y, X Z$, and $Y Z$ panels. Arrows in gray with the diamond symbol represent projections of the eigenvectors associated with the current sheet normal.

system spanned by the set of eigenvectors. The direction of the model magnetic field is associated with the eigenvector for the largest eigenvalue $\boldsymbol{e}_{1}$ (in the direction to the principal component of the magnetic field). The direction of the current sheet normal is associated with one of the remaining eigenvectors pointing closer to the north-south direction (GSM- $Z$ direction), $\boldsymbol{e}_{2}$. It should be noted that the use of a Harris current sheet implies that the model magnetic field is of a onedimensional nature embedded in three dimensions; the field is perpendicular in the direction perpendicular to $\boldsymbol{e}_{2}$ and the current sheet extends infinitely in the direction to $\boldsymbol{e}_{1}$. Construction of the Harris model is onedimensional, which in principle brings about degeneration of eigenvalues. In practice, however, if there is fluctuation or disturbance in the current sheet system, the degeneration may be resolved. On the presented time interval the three eigenvalues are separated from one another by factors 10 to 100, and establishing a reference frame based on the eigenvectors is valid. Furthermore, we impose the weak constraint that the eigenvector closest to the GSM- $Z$ direction represents the current sheet normal. The estimated normal direction has offset angles from GSM- $Z$ by about 30 degrees on average. Figure 2 displays the time snapshot of the four magnetic field vectors (in black) and the direction of the eigenvector $\boldsymbol{e}_{2}$ (identified as the current sheet normal) projected onto the three planes ( $X Y$, $X Z$, and $Y Z$ ) of the GSM coordinate system. The magnetic field data are averaged over spacecraft spin at about 11:29:04 UT. Three Cluster spacecraft (1, 2, and 4) are in the northern part of magnetotail and observe the magnetic field primarily in the sunward direction. Cluster 3 completes the nearly regular tetrahedron and is located close to the geomagnetic equator (the GSM$Z$ coordinate is small), measuring only weak magnetic fields. The current sheet normal direction (in gray) is primarily aligned with the north-south (GSM-Z) direction.
2. Estimate of current sheet distance and thickness. The signal power for fitting by the Harris current sheet is obtained by the minimum variance estimator as

$P(\ell, d)=\left[\boldsymbol{h}^{\mathrm{t}}(\ell, d)\left[\boldsymbol{s} \boldsymbol{s}^{\mathrm{t}}\right]^{-1} \boldsymbol{h}(\ell, d)\right]^{-1}$,

where $\boldsymbol{h}$ denotes the shape vector consisting of the model magnetic field in the direction of $\boldsymbol{e}_{1}$ at the position of spacecraft $i$, which is $h_{i}=\tanh \left(\frac{\ell+\Delta \ell_{i}}{d}\right)$. Here $\ell+\Delta \ell_{i}$ denotes the distance of each spacecraft from the current sheet. The symbol $s$ is the measurement vector consisting of the component of the measured field in the same direction, $s_{i}=\boldsymbol{B}_{i} \cdot \boldsymbol{e}_{1}$. The advantage of the minimum variance estimator is that it is mathematically optimized to suppress the deviation between the shape vector and the measurement vector when the shape vector does not match the measurement vector. The estimator provides a better contrast in the output signal power between signal and noise than the conventional direct covariance method $|\boldsymbol{h} \cdot \boldsymbol{s}|^{2}$ (which often gives a flatter distribution of the signal power in the parameter space). Signal power is determined in the parameter space spanned by the distance $\ell$ and the thickness $d$ by looking for a peak in that space. The analysis method was also tested using synthetic data, and the ability to identify the parameter set using only four-point magnetic field data had already been proved prior to applying it to the Cluster data. From the magnetic field sampling at 11:29:04 UT, a peak has been identified at distance $\ell=5500 \mathrm{~km}$ and thickness $d=800 \mathrm{~km}$ (Fig. 3).

3. Evolution analysis. The above procedures are repeated at each time step from 10:00 UT to 14:00 UT. By doing so, the parameter set of the current sheet model is obtained as a function of time (Fig. 1, bottom two panels). The distance becomes smaller from $7000 \mathrm{~km}$ down to $1000 \mathrm{~km}$ at about 12:30 UT. After reaching the minimum, the distance $\ell$ changes sign (not shown in the plot) and increases again. The time of closest 


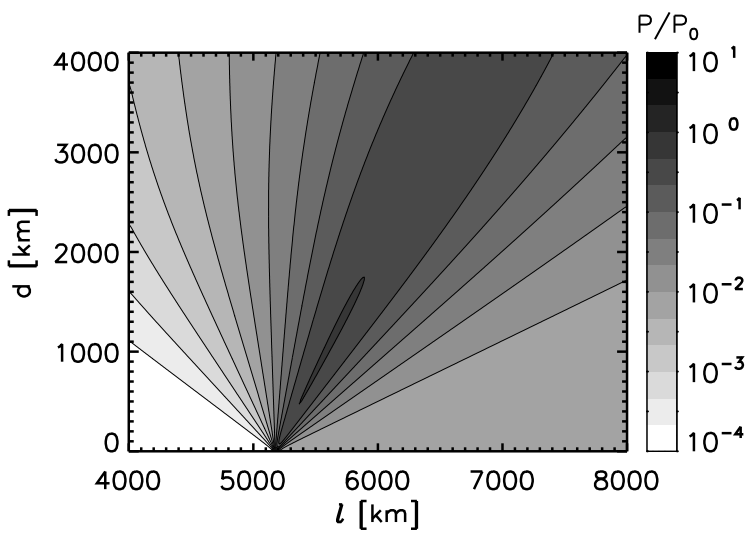

Fig. 3. Signal power (normalized to unity) of the minimum variance estimator in the parameter space spanned by the distance to the current sheet $\ell$ and the thickness $d$ derived from the four-point magnetic field measurements at 11:29:04 UT (cf. Fig. 2).

approach agrees with the sharp transition in the time series at about 12:30 UT. The current sheet thickness exhibits a rather broad distribution in the range, from several hundred $\mathrm{km}$ to $8000 \mathrm{~km}$. Around the closest approach (12:30-13:00 UT), the thickness was not determined uniquely, but the signal power distribution diverges in the parameter space as the thickness parameter $d$ is increased. After 13:00 UT, the thickness exhibits two distinct populations, one close to $1000 \mathrm{~km}$ and the other between 4000 and $7000 \mathrm{~km}$. To visualize the statistics of current sheet thickness, the histogram presented in Fig. 4 is normalized to the local proton gyroradius. The thickness is distributed from the proton gyroradius (or smaller) up to 20 gyroradii. The histogram is found to be fitted reasonably by three Gaussian distributions: the major population is approximated by a Gaussian with center $\left\langle d / r_{\mathrm{g}}\right\rangle=1.4$ and width 0.5 , the second population with $\left\langle d / r_{\mathrm{g}}\right\rangle=3.1$ and width 0.9 , and the third population with $\left\langle d / r_{\mathrm{g}}\right\rangle=$ 9.3 and width 3.5 , respectively. Large variation in the thickness distribution agrees with the recent statistical study by Rong et al. (2011). The applicability of multiple Gaussian fitting supports the notion of embedded current sheet structure as proposed by Petrukovich et al. (2011).

\section{Discussion and conclusions}

The presented analysis agrees with earlier studies that the current sheet thickness can be of the order of a proton gyroradius (e.g., Runov et al., 2006). The distribution of the thickness is rather broad, stretching up to 20 gyroradii, but shows three peaks in support of the interpretation as the embedded current sheet structure. The method is based on sensing magnetic field gradients. Since the method only assumes a local

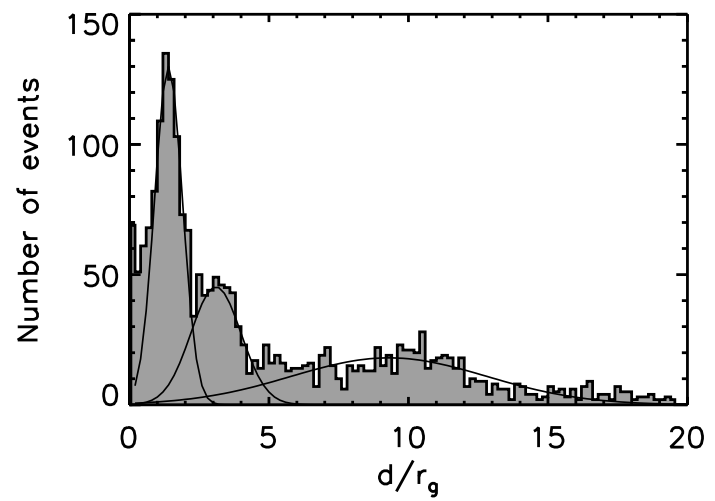

Fig. 4. Histogram of determined current sheet thickness $d$ normalized to the gyroradius of thermal protons $r_{\mathrm{g}}$. The dashed lines represent fitting with multiple Gaussian distributions.

Harris-type current sheet, it can readily be applied to Cluster magnetotail crossings under various conditions, e.g., tetrahedral formation and separation from 200 to $10000 \mathrm{~km}$, as well as different phases of solar wind and substorm activity.

The distance can be estimated during the whole time interval, but the thickness cannot be determined uniquely when the spacecraft are too close to the current sheet (around 12:30-13:00 UT). The presented method works better when the spacecraft are outside the current sheet, in contrast to the curlometer technique (e.g., Dunlop et al., 2002), which evaluates the current density through the tetrahedron. Our method uses only spatial information to estimate the geometry. It is complementary to the discontinuity analyzer (e.g., Dunlop and Balogh, 2005) that associates the timing information with the geometry and the motion. Our method uses the least-square method, as in the gradient computation (De Keyser, 2008; Hamrin et al., 2008), but minimization is applied to the deviation from the hyperbolic tangent profile of the Harris model. In contrast, the least-square gradient computation minimizes higher-order terms in Taylor expansion.

We used the eigenvectors to establish the reference frame of analysis. Other choices of reference frames are possible. For example, the GSM- $Z$ direction may be used, which can be implemented simply and which is fixed globally. This choice, however, neglects the effect of tilted current sheets (Rong et al., 2011) due to the finite dawn-dusk component of interplanetary magnetic field (IMF- $B_{y}$ ), plasma instability (kink mode), or tail-flapping. Another approach is to find the direction of the maximum field gradient (which is suitable for studying magnetopause current structures), or we do not use any preferred direction for the current sheet normal, and survey the larger parameter space by introducing the direction of the model current sheet as an additional free parameter. Our analysis method assumes a Harris current sheet, which is one-dimensional. Since the current sheet normal direction is not always known a priori, the tetrahedral configuration should be nearly regular for the equal sensitivity in various 
directions. If the normal direction is known, the method is applicable to a number of other sensors, either smaller or larger. The sensitivity becomes better if more sensors are used in the analysis.

To conclude, as Cluster samples the magnetic field at four points in tetrahedral formation, it is possible to estimate the current sheet thickness and distance directly, without associating time series data with spatial structure. A trade-off factor should be noted here. The method is independent of temporal evolution, as the spatial structure is determined from a snapshot of magnetic field samples, and the method can be used as a means of tracking the dynamical evolution of the current sheet structure. In turn, the method depends on the model and is computationally demanding, as a fitting procedure must be performed in a parameter space. The more dimensions the parameter space has, the higher the computational load needed to obtain the optimal values of the parameter set. The current sheet model may be analytical as well as numerical. Assumption of a Harris-type current sheet was made in the present analysis, as it can be implemented conveniently and, furthermore, the computational load is moderate. In reality, however, a non-Harris-type sheet (Nakamura et al., 2002; Asano et al., 2005) or higher-order structures such as deformation or multi-layers need to be taken into account and integrated into the model by adding more parameters.

Acknowledgements. This research was partly supported by the Austrian Science Fund FWF I429-N16 and I23862-N16.

Topical Editor L. Blomberg thanks J. De Keyser for his help in evaluating this paper.

\section{References}

Asano, Y., Nakamura, R., Baumjohann, W., Runov, A., Vőrős, Z., Volwerk, M., Zhang, T. L., Balogh, A., Klecker, B., and Rème, H.: How typical are atypical current sheets?, Geophys. Res. Lett., 32, L03108, doi:10.1029/2004GL021834, 2005.

Balogh, A., Carr, C. M., Acuña, M. H., Dunlop, M. W., Beek, T. J., Brown, P., Fornacon, K.-H., Georgescu, E., Glassmeier, K.H., Harris, J., Musmann, G., Oddy, T., and Schwingenschuh, K.: The Cluster Magnetic Field Investigation: overview of in-flight performance and initial results, Ann. Geophys., 19, 1207-1217, doi:10.5194/angeo-19-1207-2001, 2001.

Baumjohann, W.: Modes of convection in the magnetotail, Phys. Plasmas, 9, 3665-3667, doi:10.1063/1.1499116, 2002.

Constantinescu, O. D., Glassmeier, K.-H., Motschmann, U., Treumann, R. A., Fornaçon, K.-H., and Fränz, M.: Plasma wave source location using CLUSTER as a spherical wave telescope, J. Geophys. Res., 111, A09221, doi:10.1029/2005JA011550, 2006.

De Keyser, J.: Least-squares multi-spacecraft gradient calculation with automatic error estimation, Ann. Geophys., 26, 3295-3316, doi:10.5194/angeo-26-3295-2008, 2008.

Dunlop, M. W. and Balogh, A.: Magnetopause current as seen by Cluster, Ann. Geophys., 23, 901-907, doi:10.5194/angeo-23901-2005, 2005.
Dunlop, M. W., Balogh, A., Glassmeier, K.-H., and Robert, P.: Four-point Cluster application of magnetic field analysis tools: The Curlometer, J. Geophys. Res., 107, 1384, doi:10.1029/2001JA005088, 2002.

Escoubet, C. P., Fehringer, M., and Goldstein, M.: Introduction: The Cluster mission, Ann. Geophys., 19, 1197-1200, doi:10.5194/angeo-19-1197-2001, 2001.

Glassmeier, K.-H., Motschmann, U., Dunlop, M., Balogh, A., Acuña, M. H., Carr, C., Musmann, G., Fornaçon, K.-H., Schweda, K., Vogt, J., Georgescu, E., and Buchert, S.: Cluster as a wave telescope - first results from the fluxgate magnetometer, Ann. Geophys., 19, 1439-1447, doi:10.5194/angeo-19-14392001, 2001.

Haaland, S. E., Sonnerup, B. U. Ö., Dunlop, M. W., Balogh, A., Georgescu, E., Hasegawa, H., Klecker, B., Paschmann, G., Puhl-Quinn, P., Rème, H., Vaith, H., and Vaivads, A.: Fourspacecraft determination of magnetopause orientation, motion and thickness: comparison with results from single-spacecraft methods, Ann. Geophys., 22, 1347-1365, doi:10.5194/angeo-221347-2004, 2004.

Hamrin, M., Rönnmark, K., Börlin, N., Vedin, J., and Vaivads, A.: GALS - Gradient Analysis by Least Squares, Ann. Geophys., 26, 3491-3499, doi:10.5194/angeo-26-3491-2008, 2008.

Harris, E. G.: On a plasma sheet separating regions of oppositely directed magnetic field, Nuovo Cimm., 23, 115-121, 1962.

Haykin, S.: Adaptive filter theory (2nd. ed.), Prentice Hall information and system science series, Prentice-Hall Inc., New Jersey, 1991.

Larson, D. and Kaufmann, R. L.: Structure of the magnetotail current sheet, J. Geophys. Res., 101, 21447-21461, 1996.

Nakamura, R., Baumjohann, W., Runov, A., Volwerk M., Zhang, T. L., Klecker, B., Bogdanova, Y., Roux, A., Balogh, A., Rème, H., Sauvaud, J. A., and Frey, H. U.: Fast flow during current sheet thinning, Geophys. Res. Lett., 20, 2140, doi:10.1029/2002GL016200, 2002.

Nakamura, R., Baumjohann, W., Runov, A., and Asano, Y.: Thin current sheets in the magnetotail observed by Cluster, Space Sci. Rev., 122, 29-38, 2006.

Ness, N. F.: The Earth's magnetic tail, J. Geophys. Res., 70, 29893005, 1965.

Petrukovich, A. A., Baumjohann, W., Nakamura, R., and Runov, A.: Formation of current density profile in tilted current sheets, Ann. Geophys., 26, 3669-3676, doi:10.5194/angeo-26-36692008, 2008.

Petrukovich, A. A., Artemyev, A. V., Malova, H. V., Popov, V. Y., Nakamura, R., and Zelenyi, L. M.: Embedded current sheets in the Earth's magnetotail, J. Geophys. Res., 116, A00125, doi:10.1029/2010JA015749, 2011.

Plaschke, F., Glassmeier, K.-H., Constantinescu, O. D., Mann, I. R., Milling, D. K., Motschmann, U., and Rae, I. J.: Statistical analysis of ground based magnetic field measurements with the field line resonance detector, Ann. Geophys., 26, 3477-3489, doi:10.5194/angeo-26-3477-2008, 2008.

Rong, Z. J., Wan, W. X., Shen, C., Li, X., Dunlop, M. W., Petrukovich, A. A., Zhang, T. L., and Lucek, E.: Statistical survey on the magnetic structure in magnetotail current sheets, J. Geophys. Res., 116, A09218, doi:10.1029/2011JA016489, 2011.

Runov, A., Sergeev, V. A., Nakamura, R., Baumjohann, W., Apatenkov, S., Asano, Y., Takada, T., Volwerk, M., Vörös, Z., 
Zhang, T. L., Sauvaud, J.-A., Rème, H., and Balogh, A.: Local structure of the magnetotail current sheet: 2001 Cluster observations, Ann. Geophys., 24, 247-262, doi:10.5194/angeo-24-2472006, 2006.

Zelenyi, L., Malova, H., Artemyev, A., Popov, V., Petrukovich, A., Delcourt, D., and Bykov, A.: Magnetotail after Geotail, Interball and Cluster: Thin current sheets, fine structure, force balance and stability, Climate and Weather of the Sun-Earth System (CAWSES), edited by: Tsuda, T., Fujii, R., Shibata, K., and Geller, M. A., pp. 121-170, Terrapub, Tokyo 2009.
Zhang, T. L., Baumjohann, W., Nakamura, R., Runov, A., Volwerk, M., Asano, Y., Vörös, Z., Eichelberger, H.-U., Sergeev, V., Shi, J. K., and Balogh, A.: A statistical survey of the magnetotail current sheet, Adv. Space Res., 38, 1834-1837, 2006. 